Onkologe 2014 · 20:6-8

DOI 10.1007/s00761-013-2556-3

Online publiziert: 22. Januar 2014

(c) Springer-Verlag Berlin Heidelberg 2014

\section{P. Vajkozcy ${ }^{1}$ W. Wick ${ }^{2} \cdot$ M. Bamberg ${ }^{3}$}

${ }^{1}$ Neurochirurgische Klinik, Charité - Universitätsmedizin Berlin

${ }^{2}$ Abteilung Neuroonkologie, Neurologische Klinik und Nationales Tumorzentrum, Universität Heidelberg

${ }^{3}$ Universitätsklinikum Tübingen

\title{
Zerebrale Metastasen
}

Liebe Kolleginnen und Kollegen,

es ist uns eine große Freude, Ihnen das Leitthemenheft "Zerebrale Metastasen“ präsentieren zu dürfen.

Hirnmetastasen haben im Vergleich zu den hirneigenen Tumoren - zumindest aus klinisch- und experimentell-neuroonkologischer Sicht - in der Vergangenheit häufig ein Schattendasein geführt.

\section{》) Hirnmetastasen sind heute die am häufigsten diagnostizierten Hirntumoren}

Lange Zeit wurde ausgeblendet, dass Hirnmetastasen heute als häufigste Hirntumoren diagnostiziert werden und in unserer Praxis oft eine größere Rolle spielen als z. B. hirneigene oder gutartige Hirntumoren. Wesentliche Gründe sind die frühzeitige Diagnostik und die verbesserte Therapie des Primärtumors sowie der extrazerebralen Metastasen.

Die Therapie der betroffenen Patienten stellt heute hohe Anforderungen an die Interdisziplinarität der Partner aus Neurochirurgie, Neurologie/Neuropsychologie, Neuroonkologie, Neuroradiologie, Neuropathologie, Medizinsicher Onkologie und Strahlentherapie. Zudem kommt die Vielzahl der durch die Identität des Primärtumors getriggerten, beteiligten Fachdisziplinen (Pulmonologie, Dermatologie, Gynäkologie etc.) hinzu. Damit ist die Behandlung dieser Patienten häufig deutlich komplexer als die klassische Gliomtherapie und benötigt daher umso mehr eine gut funktionierende und von allen Beteiligten tatsächlich praktizierte Tumorzentrumsstruktur.
Die vorliegende Ausgabe von Der Onkologe hat zum Ziel, diesem Ruf nach Interdisziplinarität und der Komplexität der Patienten mit Hirnmetastasen gerecht zu werden. Die Beiträge verdeutlichen überdies die zahlreichen Fortschritte im Bereich der Grundlagenwissenschaft, bildgebenden Diagnostik, personalisierten Therapieentscheidung und Komplikationsreduktion aufgrund besserer Planung und minimal-invasiver Therapieansätze sowie neuer, jüngst etablierter und noch experimenteller Therapiekonzepte der vergangenen Jahre.

In ihrem Beitrag zu den grundlagenwissenschaftlichen Aspekten der Hirnmetastasierung geben Winkler $u$. Wick einen sehr guten Überblick über die aktuellen spannenden Fragestellungen auf diesem Gebiet. Im Zentrum steht der mehrschrittige Prozess der Hirnmetastasierung, wobei die zugrunde liegenden Mechanismen des Homing einzelner Tumorzellen in das Gehirn, der Adhäsion der Tumorzellen an den Endothelzellen, der Tumorzellextravasation, der perivaskulären Nischenbildung sowie der Kolonialisierung und soliden Metatasenbildung noch unklar bleiben. Jüngste intravitale, videomikroskopische und konfokalmikroskopische Zweiphotonenverfahren erlauben einen neuen Einblick in diesen Prozess. Die individualisierte und personalisierte Therapie von Hirnmetastasen beginnt mit einer bildgebenden Diagnostik ,Stateof-the-Art". Engelhorn u. Dörfler geben in ihrem Beitrag einen Überblick über die aktuelle Wertigkeit der Schnittbilddiagnostik und der technischen Details zur Optimierung des bildgebenden Hirnmetastasennachweises. Neben der klassischen radiologischen Diagnostik spielen funktionelle MRT-Techniken eine zunehmend größere Bedeutung. Diese helfen zum einen, wichtige differenzialdiagnostische Überlegungen auszuschließen, zum anderen dienen sie aber auch der präoperativen Funktionslokalisierung, die für die späteren lokalen Therapiemaßnahmen mitunter von großer Bedeutung sein können.

In der lokalen Kontrolle symptomatischer Hirnmetastasen kommt nach wie vor der mikrochirurgischen Resektion eine zentrale Bedeutung zu. Dies wird in dem Beitrag von Schackert $u$. Jurath detailliert dargelegt. Der Beitrag arbeitet anhand aktueller Studienergebnisse die Rolle der Operation in der Therapie von Hirnmetastasen auf und geht unter anderem auch auf die jüngsten Ergebnisse zur Bedeutung der Resektionstechnik für das lokale Rezidiv ein. Kontrovers wird die Operation multipler Hirnmetastasen diskutiert. Auch hier geht der Beitrag sehr illustrativ und praktisch relevant auf das Thema ein.

Eine Besonderheit bei der Operation von Hirnmetastasen stellt die Priorität des neurologischen Funktionserhalts dar. Im Beitrag von Krieg at al. werden die Möglichkeiten der präoperativen Funktionslokalisierungen und intraoperativen Funktionsüberwachung im Detail beleuchtet. Von besonderer Bedeutung ist hierbei die jüngste Entwicklung der transkraniellen Magnetstimulation zur präzisen präoperativen Identifizierung von motorischen oder sprachrelevanten Arealen. Aber auch die elektrophysiologischen Methoden mit der kortikalen und subkortikalen Stimulation als zentraler Bestandteil des intraoperativen Monitoring werden im Detail erläutert. 
Hier steht eine Anzeige.

黛 Springer 
Neben der Operation kommt der klassischen Strahlentherapie sowohl in der Lokalkontrolle als auch in der Ganzhirnbestrahlung eine zentrale Bedeutung zu. Dunst $u$. Rades gehen diesbezüglich auf die aktuellen Evidenzen in der Anwendung der klassischen Strahlentherapie zur primären und adjuvanten Therapie von Hirnmetastasen ein. Es wird klar, dass in Abhängigkeit vom Kontext die lokale Nachbestrahlung nach Operation oder die Ganzhirnbestrahlung primär oder nach Operation von zentraler Bedeutung zur Verhinderung weiterer Rezidive sind. Es wird aber auch klar, dass die Entscheidung zur Ganzhirnbestrahlung aufgrund möglicher neuropsychologischer Nebenwirkungen im Einzelfall stets mit Bedacht getroffen werden sollte.

Eine Alternative zur klassischen Strahlentherapie ist die Radiochirurgie. Im Beitrag von Kufeld et al. werden die Vorteile und die Möglichkeiten der lokalen Strahlentherapie mittels der Radiochirurgie beleuchtet. Der Radiochirurgie kommt hierbei als Konkurrenzverfahren zur mikrochirurgischen Resektion ein besonderer Stellenwert zu. In ihrer Anwendung ist die Radiochirurgie sehr stark von der Tumorgröße abhängig. Auf der anderen Seite handelt es sich um ein effektives Verfahren mit geringer Belastung für den $\mathrm{Pa}$ tienten.

\section{ل) Die Prognose für Patienten mit Hirnmetastasen solider Tumoren bleibt ungünstig}

Abschließend gehen Korfel u. Schlegel mit ihrem Beitrag auf die Wertigkeit der Chemotherapie bei Hirnmetastasen ein. Sie kommen zu dem Schluss, dass eine entscheidende Verbesserung der Prognose von Patienten mit Hirnmetastasen durch den Einsatz systemischer Therapien bisher nicht erreicht worden ist. Sie stellen aber heraus, dass die Indikation zur Chemotherapie sehr stark vom Primärtumor abhängig ist. So repräsentieren das kleinzellige Bronchialkarzinom oder Keimzelltumoren chemosensible Tumoren, bei denen die Chemotherapie als primäre Therapie eingesetzt werden sollte, ggf. in Kombination mit der klassischen Radiotherapie. Bei anderen Primärtumor- formen muss die Entscheidung zur Chemotherapie in Abhängigkeit von der Tumordissemination und vom Allgemeinzustand des Patienten im interdisziplinären Kontext entschieden werden.

Die Beiträge zeigen aber auch, dass in der Zukunft noch viel für die Tumorpatienten getan werden muss. Während man heute signifikante Verbesserungen für die lokale Tumorkontrolle bei Erhalt einer guten Lebensqualität verzeichnen kann, bleibt die Prognose für Patienten mit Hirnmetastasen solider Tumoren trotz aller Fortschritte der letzten Jahre mit wenigen Ausnahmen - bei einem medianen Überleben von nur wenigen Monaten - ungünstig. Prognosebestimmend ist weiterhin die systemische Tumormanifestation, die mit derzeitigen Therapiestrategien oft nicht mehr behandelt werden kann. Diese Tatsache stärkt den Ruf nach einem weiteren Ausbau der experimentellen und klinischen Forschungsaktivitäten und einer Weiterentwicklung interdisziplinärer Netzwerke zum Wohl dieser komplexen Patientengruppe.

Für die Schriftleiter des Schwerpunktthemas
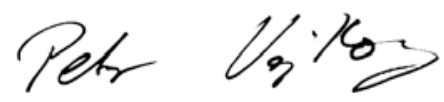

P. Vajkoczy

Für die Herausgeber
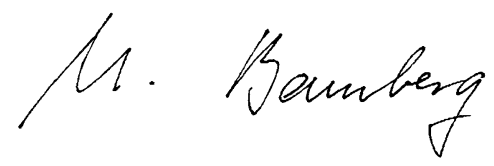

M. Bamberg

\section{Korrespondenzadresse}

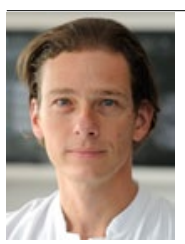

Prof. Dr. P. Vajkozcy

Neurochirurgische Klinik, Charité - Universitätsmedizin Berlin

Augustenburger Platz 1, 13353 Berlin peter.vajkoczy@charite.de

\section{Nachlese und Ausblick: Die Krebskongresse ASH und DKK im Fokus}

Hämatologie-Experten aus aller Welt trafen sich im Dezember 2013 in New Orleans zum 55. Annual Meeting der American Society of Hematology (ASH). Auf dem Internetportal der Deutschen Krebsgesellschaft (DKG) stellen deutsche Kongressteilnehmer im Rahmen von Expertenrunden und -interviews die wichtigsten Ergebnisse vor. Ein zentrales Thema der „Expertenrunde MDS, AML und CML" ist die systematische Entdeckung von Driver-Mutationen, die dem myelodysplastischen Syndrom (MDS) zugrunde liegen. Hier konnte eine Vielzahl von Mutationen identifiziert werden, die sich in vier Gruppen einteilen lassen: Mutationen von Transkriptionsfaktoren, Signalwegen und des Splicing Apparatus sowie epigenetische Mutationen. Bei über $80 \%$ der Patienten liegt mindestens eine Mutation, die als Driver-Mutation charakterisiert ist, vor. Der Stellenwert für Therapieansprechen und Prognose der verschiedenen Mutationen muss jedoch noch geklärt werden.

Die vollständige Aufzeichnung aller Expertenrunden, Interviews und Berichte zum ASH finden interessierte Ärzte unter www.krebsgesellschaft.de/ash2013. Außerdem online: ein Ausblick auf das Programm des Deutschen Krebskongresses 2014, der seine bisherige Struktur fachbezogener Veranstaltungen erstmals aufbrechen wird. Wie DKK-Kongresspräsident Prof. Michael Hallek im DKG-Gespräch erläutert, sollen die Sitzungen stattdessen grundsätzlich interdisziplinären Charakter haben. In den Arbeitsgemeinschaften der DKG sei dieses Konzept mit großem Zuspruch aufgenommen und mitgestaltet worden. Interviews mit den Vorsitzenden der Plenarsitzungen geben unter www.krebsgesellschaft.de/dkk2014 Einblicke in die zentralen Themen.

Quelle: Deutsche Krebsgesellschaft (DKG), www.krebsgesellschaft.de 